\title{
Call for Papers
}

\section{Geriatric Medicine and End-of-Life Care Deadline June 1, 2013}

The Linacre Quarterly is preparing a special issue on geriatric medicine and end-of-life care and is requesting manuscripts that are focused on these areas. The manuscripts could address, but are not limited to:

- Medical aspects of geriatric medicine and end-of-life care, including clinical decision making, innovative models of care delivery, quality improvement, impact and cost effectiveness

- Scientific aspects of geriatric medicine and end-of-life care, especially advanced disease, multi-morbidity, acute and chronic illness

- Ethics and moral/theological aspects of geriatric medicine and end-of-life care including medically assisted nutrition and hydration, POLST orders, living wills and withdrawal of life sustaining treatment

- Compelling and informative case reports illustrating authentic Catholic practice of geriatric medicine and end-of-life care, and perhaps highlighting controversial issues

- Reviews of relevant books, essays, professional development, history, and perspectives on geriatrics and end-of-life care

- Spiritual aspects of geriatric medicine and end-of-life care including the effects of end-of-life decisions on family members.

The goal of this special issue is to educate physicians and health-care professionals regarding the medical, scientific, ethical, and spiritual aspects of geriatric medicine and end-of-life care. Primary research articles in this area are encouraged. We also encourage articles in which physicians and ethicists are paired to add depth to the medical/scientific analyses with the ethical analyses of specific issues. If you are interested in working with a physician or ethicist, and would like assistance in identifying one, resources are available.

The deadline is June 1, 2013. If you have any questions, please contact us at LQ@cathmed.org. Visit our website for additional information, http://www.cathmed.org/issues_resources/linacre_quarterly/. 